\title{
LES ETINCELLES DE LA REVOLUTION DE 25 JANVIER D'APRES LES CHRONIQUES DE LA REVOLUTION EGYPTIENNE DE ALAA EL-ASWANY ET L'EgYPTE DE TAHRIR DE ClaUde GUIBAL ET TANGI SALAUN
}

\author{
ETUDE PRÉSENTÉE PAR \\ RANIA EZZ EL ARAB \\ Maître de conférences \\ Département de langue et de littérature françaises \\ Faculté des Lettres \\ Université d'Alexandrie
}

مجلة الارسات التربوية والاسانية ــكلية التربية ـ جامعة دمنهور المجلد الخامس العدد (4)- الجزء الثالث- لسنة 2013 
LES ETINCELLES DE LA REVOLUTION DE 25 JANVIER D'APRES LES CHRONIQUES DE LA REVOLUTION EGYPTIENNE DE ALAA EL-ASWANY ET L'EgYPTE de TAHRIR de ClaUde GUIBAL ET TANGI SALAUN

\section{d. Rania Ezz El Arab}

Depuis longtemps, l'étude de l'Egypte tente de nombreux écrivains de toutes les nationalités. Pendant les dix dernières années du mandat de Hosni Moubarak, l'intérêt accordé à notre pays augmente, les essais et les ouvrages qui en traitent se multiplient. Pour son importance régionale et mondiale peut-être, mais surtout, parce que l'Egypte devint, en cette dernière décennie, «un cas » incompréhensible voire un phénomène. Portant un regard bref sur l'Egypte, la personne la moins avisée au monde se voit poser cette question: pourquoi l'Egypte régresse alors que le monde progresse ${ }^{1}$ Dotée de quelques informations, une question plus pertinente lui vient aussitôt à l'esprit : pourquoi les Egyptiens ne se soulèvent-ils pas ? ${ }^{2}$

Question choquante car elle admet à la fois une situation désastreuse qui appelle à la révolte en même temps qu'elle lance une critique aux Egyptiens qui acceptent de vivre dans de telles conditions et pourtant restent muets, passifs. Question à laquelle il est difficile de répondre puisqu'elle fut le sujet de plusieurs études élaborées par de grands penseurs et écrivains égyptiens ${ }^{3}$ avant de trouver une réponse partielle mais non définitive dans le déclenchement effectif de la révolution du 25 janvier.

Dans une tentative d'examiner de près les étincelles du « phénomène révolutionnaire ${ }^{4} \gg$ égyptien aussi bien que les coulisses de la révolution égyptienne du 25 janvier, nous avons eu recours à deux ouvrages:le premier, l'Egypte de Tahrir, écrit par deux Français intimement liés à

\footnotetext{
${ }^{1}$ Question empruntée à l'un des articles de l'écrivain Alaa El-Aswani, Chroniques de la Révolution égyptienne, Paris, Editions Actes Sud. Traduit de l'arabe et préfacé par Gilles Gauthier, p.53.

${ }^{2}$ Alaa El Aswani, Chroniques de la révolution, p.9.

${ }^{3}$ Dont bien sûr Alaa El Aswani mais aussi Galal Amine.

${ }^{4}$ Puisqu'aujourd'hui nous savons qu'il s'agit de plus d'une révolution ou de plus d'une vague révolutionnaire.
} 
l'actualité égyptienne ${ }^{5}$ et le second, Chroniques de la

révolution égyptienne écrit par «le plus célèbre des écrivains égyptiens contemporains » tel que le présente les éditeurs mêmes d'Actes Sud, responsables de traduire son œuvre dans la langue française au fur et à mesure de l'apparition de celle-ci. Le premier est publié aux éditions du Seuil, en mai 2011, le deuxième, composé d'une cinquantaine d'articles écrits quelques mois avant la révolution, en novembre de la même année.

Les deux ouvrages diffèrent du point de vue structure. Le premier, l'Egypte de Tahrir est une sorte de radiographie vivante de l'Egypte au moment où celle-ci s'apprête à changer d'ère:il est basé donc sur des rencontres et des témoignages surtout en ce qui concerne les premiers chapitres. Le second Chroniques de la révolution égyptienne n'est pas un ouvrage à proprement parler ${ }^{6}$.Il est composé d'articles que le traducteur français Gilles Gauthier a décidé de classer en trois grandes catégories. Ceux de la première partie sont groupés sous le titre de « La Présidence et la succession », la deuxième sous le titre de «Le peuple et la justice sociale »et la troisième sous le titre «Liberté de parole et répression politique $\gg$.On y trouve donc parfois des redites ou des répétitions qui ne nuisent aucunement à la fluidité de la lecture puisque ces redites sont ou bien citées dans un contexte différent, ou bien présentées sous un angle différent ou alors tout au moins, citées d'une manière différente.

Les points communs sont évidemment très nombreux, puisque les deux ouvrages traitent du même sujet, mais la plupart du temps il s'agit d'une approche différente. Parfois les auteurs français s'attardent sur un point que l'écrivain égyptien met un peu au ban et parfois c'est l'inverse. Pour ce qui est surtout de la deuxième partie de L'Egypte de Tahrir l'analyse est plutôt verticale : chacune des composantes de la place Tahrir (qui devient symbole de l'Egypte) est étudiée à part, les Frères musulmans, les Gama'at Islamia, les coptes et ainsi de suite.

${ }^{5}$ Claude Guibel, 37 ans, est journaliste, spécialiste du Proche-Orient. Elle vit au Caire depuis 1997 et travaille pour Radio France et Libération depuis 2000. Tangi Salaun, 38 ans, est le correspondant du Figaro, de L'Express et de RTL en Egypte. Il couvre l'actualité proche-orientale depuis quatorze ans.

${ }^{6}$ Des cent quatre-vingt-trois articles parus dans la presse égyptienne (pour l'essentiel dans les quotidiens Shorouk et El Masri el Yom) au cours des trois dernières années qui ont précédé la révolution, quarante-cinq articles qui ont été jugés les plus représentatifs font l'objet principal de cette traduction. Cinq articles jugés d'actualité furent ajoutés par la suite par l'éditeur. Un ensemble de cinquante articles.

doi :10.12816/0004234 
Dans cette étude, nous ne nous proposons point de traiter l'entité des thèmes qui existent dans les deux ouvrages, un travail semblable aurait été colossal, vu le nombre de pages des deux ouvrages et la richesse de leurs contenus. Nous ne comptons même pas analyser exhaustivement toutes les causes de la révolution (bien que dans un premier temps nous ayons eu l'intention). Nous avons décidé, après une longue réflexion, de nous limiter à l'étude des étincelles de la révolution selon chacun des écrivains pour pouvoir comparer librement les différentes facettes du sujet traité tellement les différentes approches nous ont passionnée.

Contrairement aux auteurs français qui commencent leur livre par la description de 1'Egypte qui dort le jour de congé ${ }^{7}$, Alaa el-Aswany commence le sien par une question perturbante qui brûle les étapes et surprend le lecteur. « Pourquoi les Egyptiens ne se soulèvent pas ? » Le lecteur s'attend à apprendre pourquoi plutôt les Egyptiens doivent se soulever !

En fait, la question de l'Egypte qui ne se soulève pas occupe profondément l'esprit d'Alaa el- Aswany plus que les causes mêmes du soulèvement. Il analyse les causes de ce mutisme et de cette apathie en plusieurs pages ; les premières, dans l'introduction. Ceci ne manque pas d'être signifiant. Sans rien dire, el-Aswany dit tout. La situation du pays est désastreuse, le mutisme est incompris, voire inadmissible. Le lecteur choqué, comprend vite ce que l'auteur insinue et se voit en un clin d'œil son complice.

A cette question, «pourquoi les Egyptiens ne se soulèvent pas ? », il y a, selon el-Aswany trois façons d'y répondre : certains pensaient que les Egyptiens ne se soulèveraient jamais. Las et soumis après de longues années de répression intolérable exercée par la machine infernale de l'Etat policier, ceux-ci étaient incapables de protester et avaient choisi bon gré malgré de rester muets. D'autres admettaient qu'une révolution était possible mais pas pour le moment. Différents facteurs devaient être responsables de son retard. La force de la répression qui effrayait la population et qui assurément calmait ses ardeurs et que la loi d'urgence, en vigueur depuis trente ans a rendue presque légale, d'une part et

${ }^{7}$ C'est le calme qui précède la tempête : nous sommes le mardi 25 janvier 2011.

599 
l'absence d'une organisation ou d'un leader ${ }^{8}$ capable de conduire les masses d'autre part. En deuxième lieu, l'extrême difficulté de la vie quotidienne qui empêche les Egyptiens de s'occuper de quoi que ce soit à part le fait de gagner leur vie. Ces pauvres Egyptiens « exclusivement occupés par leur pain quotidien et par la recherche de solutions individuelles à la crise ».(CRE,10). En troisième lieu, l'exil que s'est imposé un grand nombre d'Egyptiens suite à l'accentuation de la répression et la pauvreté. Plutôt deux genres d'exil puisqu'il ne s'agit pas chez Alaa El-Aswany d'un exil uniquement vers les Etats pétroliers du Golfe «pour y travailler dans des conditions généralement humiliantes » $(\mathrm{CRE}, 10)$ et revenir chez eux avec « de quoi s'assurer une vie acceptable $\gg(\mathrm{CRE}, 10)$ ce qu'el-Aswany appelle un exil géographique. Mais il s'agit aussi d'un autre exil auquel l'auteur donne le nom d'exil historique. Ceux qui ont choisi ce refuge «s'accrochaient au passé et vivaient en imagination dans ce qu'ils considéraient comme l'âge d'or de l'islam » (CRE,10). Ces exilés «portaient des tuniques blanches et se laissaient pousser la barbe. Ils s'étaient donné le nom d'ancêtres et fuyaient dans les gloires du passé la dure réalité » (CRE, 10). Le plus dangereux dans la pensée de ses exilés historiques, c'est que l'interprétation wahhabite ${ }^{9}$ de l'islam dont ils sont convaincus ordonne au musulman l'obéissance à celui qui gouverne «aussi oppressif et corrompu soit-il ». (CRE,11).

D'autres encore, une troisième catégorie de personnes dont Alaa elAswany, postulaient que les Egyptiens n'étaient point des gens las ni soumis, le nombre de révolutions qu'ils ont faites au cours du XXe siècle par rapport aux occidentaux en témoignent. Mais les Egyptiens

\footnotetext{
${ }^{8}$ Pour le moment, Alaa el-Aswany parle uniquement d'organisation et non pas de leader, mais le lecteur comprend par la suite qu'il peut aussi s'agir d'un chef charismatique capable de conduire les masses quand il voit l'auteur décrire le rassemblement de la foule autour de Mohamed el Baradei.

${ }^{9}$ Le lecteur arabe sait que 'islam Sunnite reconnaît quatre écoles de jurisprudence religieuse : le malékisme, le chaféisme, le hanafisme et le hanbalisme. C'est au sein de cette dernière école, considérée comme la plus stricte et littéraliste qu'est née au début du XVIIIe siècle, dans la péninsule arabique, une tendance encore plus rigoriste, le wahhabisme. Allié depuis le début à la dynastie des Al Saoud, le wahhabisme est devenu la doctrine officielle de l'Arabie saoudite. Le salafisme, lui, se définit comme un mouvement de renaissance de l'Islam, par un retour à la foi des origines. Même si leur histoire n'est pas exactement la même, ces deux conceptions intransigeantes de la religion se recoupent largement pour être l'aliment spirituel et la source d'inspiration de vastes secteurs largement abreuvés par l'argent - public ou privé - provenant de la rente pétrolière. (Alaa el-Aswany, p.11).
}

doi :10.12816/0004234 
«avaient cette particularité d'être moins portés à la violence et plus favorables aux solutions médianes » (CRE,11).Ceci peut-être parce qu'ils sont originaires d'une vieille nation « dont l'histoire s'étendait sur cinq mille ans » $(\mathrm{CRE}, 11)$ ce qui les dotait d'une certaine sagesse, " la sagesse des vieillards » (CRE,11) qui les poussaient à « se tenir, dans la mesure du possible, à l'écart des problèmes pour se contenter de vivre et d'élever leurs enfants »(CRE,11). Alaa el-Aswany, lui, était convaincu que la révolution ne tardera pas à venir, bien au contraire, il savait qu'elle approchait et qu'elle allait être imminente. «Pas un jour (il) n'a cessé d'avoir foi dans le peuple » dit-il de lui-même (CRE,11), pas un jour il n'était d'accord avec ses nombreux amis qui l'«accusaient» (CRE,11) d'être optimiste et romantique.Alaa el-Aswany voyait le peuple égyptien à l'image du chameau. Comme lui, « il pouvait supporter les coups, les humiliations, et la faim pendant une longue période » mais une fois convaincu qu'il n'y avait d'autres solutions que la révolution, il se révolterait, et d'une façon «soudaine » $(\mathrm{CRE}, 11)$ et avec « une force irrépressible » $(\mathrm{CRE}, 11)$.

Pour el-Aswany, ce qui manquait aux Egyptiens en ce moment c'était d'être convaincus qu'il n'y avait d'autres solutions que la révolution. Selon lui, il était temps qu'ils comprennent que«les adjurations et les requêtes pleines de fioritures ne servaient (plus) à rien » et que «pour avoir nos droits, il n'y a ni intérêt ni utilité à implorer le régime qui ne répondra pas à notre requête » $(\mathrm{CRE}, 64)$ et el-Aswany de conclure : «les droits ne s'octroient pas, ils s'arrachent » (CRE,62). Cette phrase, est d'une grande importance chez l'auteur : il la répète au moins deux fois telle quelle dans au moins deux articles différents.

Cette question : pourquoi les Egyptiens ne se soulèvent pas ? Guibal et Salaun ne la posent même pas. Ils y répondent implicitement par quelques mots mais des mots pertinents. Ceci lorsqu'ils parlent de deux des activistes, des déclencheurs de la révolution Israa Abdel Fattah et Ahmed Maher, tous deux dans un premier temps, membres du Mouvement 6 avril avant la lettre.

Quand ils entendent que les hommes de Mahalla envisagent une grève le 6 avril 2008 pour protester contre leurs salaires médiocres, les deux jeunes gens compatissent et décident de les aider. Israa Abdel Fattah appelle donc à travers son ordinateur à une grève générale. Aux trois cent invitations lancées, trois mille personnes répondent 
favorablement. Mais Guibal et Salaun commentent: «mais (les deux activistes)savent :entre un clic d'appréciation et une participation effective à un événement, il y a un monde. Un monde de peur, un monde de fatalisme, de résignation » et le duo français d'ajouter : «Et l'Egypte, blaguent bien souvent les Egyptiens eux-mêmes, est la terre IBM. IBM pour Inch' Allah (à la grâce de Dieu), Boukra (demain), Maalesh (tant pis) ${ }^{10}$. La terre du 'un jour peut-être'» ET,72). Lâcheté, passivité, soumission aux yeux des auteurs français sont les causes du mutisme de l'Egypte qui se laissait aller sans broncher. Trois adjectifs haïssables que les auteurs réduisent en trois mots, les mots les plus utilisés dans notre vie quotidienne.

Cette vie quotidienne des Egyptiens a de quoi provoquer les auteurs. Que ce soit Guibal ou alors Salaun ou même Alaa el-Aswany, tous pensent que c'est une vie qui ne mérite point d'être appelée ainsi. Les écrivains français, à travers les épisodes qu'ils ne cessent de puiser dans la vie quotidienne des Egyptiens ${ }^{11}$, sans le dire d'ailleurs explicitement, semblent ancrer le lecteur, petit à petit, dans la conviction que la révolte du peuple égyptien est une question de temps. Quant à Alaa El Aswany, connu pour sa franchise parfois un peu gênante, il n'éprouve aucun inconvénient à le dire, ouvertement, dès les premières lignes de l'introduction de son livre juste après avoir posé sa question et avant d'y répondre: «Toutes les conditions d'une révolution se trouvaient pourtant réunies » (CRE,9).

Ces conditions tout le monde les connaît. Les plus récentes du point de vue chronologique ont servi d'étincelles à la révolution. Pour Guibal et Salaun, «l'étincelle fatale qui conduisit à la fin du régime d'Hosni Moubarak »(ET,80)fut l'affaire de Khaled Said.Cette histoire dramatique revêt une importance singulière chez les trois écrivains, qui lui consacrent des pages, la racontent en détails et la commentent longuement. Selon les auteurs français, l'histoire aurait pu rester au stade d'" une sale bavure, une affaire qui tourne mal, et que l'on commenterait à voix basse, honteux et terrifié d'éventuelles représailles » $(\mathrm{ET}, 80)$ mais refusant l'accès de la famille du jeune homme à la morgue pour récupérer son corps, dire qu'il est mort étouffé

${ }^{1010}$ Le mot maalesh, Guibal et Salaun le commentent aussi à la page 46 du même ouvrage. "Vocable essentiel du dialecte égyptien, à la fois synonyme de 'tant pis', 'c'est comme ça', 'ce n'est pas grave', plombé de fatalisme, utilisable aux fins les plus futiles comme les plus graves, ponctuant toutes les conversations. »

${ }^{11}$ Comme le récit des «tomates folles » que nous citerons plus tard en discutant du marasme économique.

doi :10.12816/0004234 
en avalant un sachet de drogue, cherchant à le dissimuler des officiers et qu'il était «un délinquant, recherché pour ne pas avoir fait son service militaire et pour possession d'arme » $(\mathrm{ET}, 80)$ c'est ceci qui était exagéré. Exagéré parce que peu vrai. Parce que le centre Nadim pour la réhabilitation des victimes de la torture fut prévenu. « Leurs chercheurs et leurs avocats, ainsi que ceux de Human Rights Watch, enquêtèrent ». Ils obtinrent le témoignage du propriétaire du cybercafé et de quelques personnes qui passaient dans la rue en ce moment-là. Tous les récits convergèrent. Et ces témoignages ne ressemblaient en rien à ce qui a été dit par les deux policiers accusés.

Le reste de l'histoire est connu. Les deux photos de Khaled Said qui circulèrent aussitôt sur Internet et qui émurent tous les Egyptiens « reflétant une triste réalité : l'Egypte fait à ses enfants ce que ne leur ont pas fait ses ennemis » (CRE, 240), de blogs en pages créés aussitôt sur Facebook, la nouvelle autopsie du corps qui confirme le résultat du premier examen médico-légal et la montée de la vague de contestation, l'opposition qui réclame la fin de la loi d'urgence et, pour la première fois, le grondement qui s'enfle et qui dépasse « les cercles habituels de la contestation », $(\mathrm{ET}, 81)$ et enfin, la création de la page «Kollena Khaled Said», «nous sommes tous Khaled Said» qui devint très rapidement la plateforme de contestation avant d'appeler «le vent de colère à souffler sur l'Egypte » $(\mathrm{ET}, 82)$.

Ce qui tendit encore plus la situation et fut plus provoquant encore pour les Egyptiens, selon les témoignages des auteurs français, ce fut cette fête de la police, que l'Etat a insisté à célébrer ce 25 janvier 2011. Ceci, malgré les abus de la police connus pour tous et bien qu'elle soit accusée d'être l'incarnation même de la répression et de la torture et bien qu'elle soit le seul responsable de la protection de ce régime autocrate. D'emblée, cette année, la célébration de la police a lieu huit mois après le crime scandaleux commis par les deux policiers, à Alexandrie, contre le jeune Khaled Said. Pourquoi ce défi au peuple, pourquoi ce désintérêt de ses sentiments ? «Indécent pensent beaucoup. Intolérable même pour certains » (ET, 11-12).

Quant à Alaa el-Aswany, il utilise l'histoire de Khaled Said pour inciter le peuple à changer son destin en lui montrant encore une fois que contrairement à ce que l'on pourrait croire, « la soumission n'est plus capable de protéger les Egyptiens contre la répression. » (CRE, 239-240). A croire el-Aswany, Khaled Said n'a jamais d'activité 
publique, il n'était membre d'aucun des mouvements qui visaient au changement de régime. Peut-être même n'avait-il jamais participé à une manifestation de sa vie. Khaled était un jeune Egyptien complètement pacifique. Pourtant, il a été frappé de la même façon que le sont les jeunes qui manifestent pour la liberté. Le destin de Khaled Said pouvait être celui de n'importe quel égyptien. C'est ainsi qu'el-Aswany incite le peuple à changer sa condition car «le seul moyen de se délivrer du chef oppressif est de lui faire face avec toutes les forces dont on dispose. » (CRE, 241).

Deuxième étincelle ${ }^{12}$ selon Guibal et Salaun: les élections législatives de novembre 2010. Ces élections, Alaa el-Aswany appelle à les boycotter et assure que ce boycott « est la position saine qu'à adoptée le peuple égyptien, et par conséquent, tous ceux qui iront voter seront en contradiction avec la volonté du peuple.»(CRE,113) La raison en est la suivante : ces élections seront «frauduleuses »(CRE,79).Guibal et Salaun sont d'accord sur ce dernier point. Ils affirment que «Personne n'a de doute sur l'issue du scrutin.» (ET,60).Pourquoi ? Parce que de nombreux indices en témoignent. Dans le passé, le régime utilisait les juges pour «couvrir»(CRE,79) la fraude électorale mais le nombre de juges refusant d'accomplir ce travail augmentait de plus en plus. Message : ils étaient de moins en moins satisfaits de la démarche du processus électoral. En cette année 2010, ce fut le régime lui-même qui décida d'abolir le contrôle judiciaire selon les modifications qui ont été apportées à la Constitution en 2007. De même, il a refusé de lever l'Etat d'exception et de réviser les listes électorales « pour en enlever les noms des personnes décédées (qui votent toujours pour le parti au pouvoir) » (CRE,110). En même temps, Amnesty International et les organisations égyptiennes des droits de l'homme « dénonçaient le climat délétère, les intimidations ${ }^{13}$, la tension » aussi bien que les restrictions imposées aux médias indépendants et la répression contre les Frères musulmans (ET, 82). Simultanément, le régime égyptien refusa toute mission d'observation internationale et «renvoya dans ses cordes l'administration américaine qui a demandé en vain au Caire d'accepter la venue d'observateurs internationaux» $\gg$ ET,82). Que faut-il comprendre?

A la veille du vote, soulignent les auteurs français, « les dirigeants du PND ne cherchent même pas à sauver les apparences. » (ET,61). Ils

${ }^{12}$ Toujours par ordre chronologique descendant donc du plus récent au plus ancien.

${ }^{13}$ Des services de sécurité.

doi :10.12816/0004234 
pronostiquent une forte progression de l'opposition laïque au détriment des Frères musulmans. Les Egyptiens comprennent que cette fois-ci ce sont les libéraux et la gauche qui vont servir d'alibi démocratique au régime.

Mais le régime de Moubarak qui s'intéresse peu à son peuple et « goûte peu les critiques » $(E T, 82)$ n'a pas d'inconvénient à continuer la comédie. La presse officielle présente la journée des élections comme « la grande fête de la démocratie » $(\mathrm{ET}, 82)$. Le régime soutient le Premier ministre Ahmed Nazif qui explique :«Nous allons prouver au monde que nous sommes capables de gérer le processus électoral d'une manière intègre » $(\mathrm{ET}, 82)$. Le ministre de l'Intérieur Habib el-Adly n'oublie pas de rappeler que «les manifestations ne font pas partie des mécanismes de la campagne électorale » et que la police «agira avec toute la fermeté nécessaire » face à quiconque « tenterait de porter atteinte à la stabilité » du processus. (ET,83).

D'accord sur les faits qu'ils racontent presque de la même manière et d'accord aussi sur le peu d'intégrité de ces élections, les conclusions que tirent Guibal et Salaun sont différentes de celles d'Alaa el-Aswany. Ce dernier dit franchement au régime que les Egyptiens sont au courant de ce qui se passe autour d'eux : «Les Egyptiens savent déjà parfaitement que les membres du parti au pouvoir remporteront la majorité des sièges au parlement et que l'élection présidentielle sera une farce qui permettra au président Moubarak de conserver le pouvoir ou d'en faire hériter son fils Gamal. »(CRE,77).

A cette conclusion à long terme, d'un pessimisme flagrant d'ailleurs, les deux auteurs français opposent des résultats plus proches en s'intéressant surtout aux répercussions de ces élections après le premier et le second tour. Répercussions chez les internautes et les activistes, véritables leaders de la révolution qui couve, les médias internationaux, la Maison Blanche. Répercussions aussi chez l'Etat, l'opposition, la commission électorale et enfin le PND. Pour ce qui est des internautes et des activistes, ils ont « conscience d'avoir remporté une petite bataille » $(E T, 83)$ car ils savent que ce scandale est en leur faveur, puisqu'il met le régime qu'ils veulent renverser à découvert devant la société internationale et montre clairement à celle-ci la décadence de la démocratie en Egypte et les abus du régime dictatorial en matière politique. Ceci est susceptible de donner une légitimité à la résistance des activistes égyptiens contre le régime de Moubarak; une légitimité 
dont ils ont besoin non pour que les pays occidentaux viennent à leur aide (et à celle de tous les jeunes égyptiens comme on le saura plus tard) ce que ces premiers refusent catégoriquement mais uniquement dans l'espoir que ces grandes puissances cessent de soutenir le régime du tyran.

Les médias internationaux prévenus des abus par Twitter «les commentent abondamment, poussant la Maison-Blanche à réagir et à faire part de sa consternation » $(\mathrm{ET}, 83)$. Les auteurs français n'oublient pas de souligner la réaction de l'Etat envers les activistes : les agents de Amn el-Dawla, la Sécurité de l'Etat essaie de les espionner surtout à travers Facebook, ils multiplient les arrestations leur prennent leur portable, leurs papiers d'identité en vue de les dissuader de leurs projets.

Quant à l'opposition, y compris les Frères musulmans, ne purent pas supporter la «mascarade » $($ ET,61). En fait, « les fraudes sont telles » $(\mathrm{ET}, 84)$ que l'opposition refuse de participer au second tour de l'élection. La commission électorale, elle, admet des «incidents mineurs » $(\mathrm{ET}, 84)$ sans conséquences sur l'ensemble du processus, elle invalide les comptes de quelques urnes.

Cependant, le PND ne se prononce qu'après les résultats scandaleux du deuxième tour qui n'a vu s'affronter que deux des candidats du PND. C'est en ce moment précis que finalement, au sein du PND, « le trouble pointe ». Devant les caméras, les membres du parti continuent à ironiser l'opposition qui s'est retirée avant le second tour mais, à croire Guibal et Salaun, «ils s'avouent dépassés par le phénomène. Ils admettent que cette situation décrédibilise l'image du PND en remettant en question sa capacité à réformer le pays, comme Gamal Moubarak, le fils du président et possible successeur du rais, s'y est engagé. » $(\mathrm{ET}, 84)$. Et Guibal et Salaun de conclure : «le déroulement du scrutin donne raison aux neufs Egyptiens sur dix qui ne se sont pas déplacés pour voter. » (ET,61).

Cependant, les faits et les conséquences liés à la fraude et que nous venons de décrire selon les trois écrivains ne forment pas l'essentiel du sujet ni pour les Français ni pour l'Egyptien. La fraude des élections est simplement le leitmotiv. Le corps du sujet étant totalement différent puisque les deux approches ne convergent point. Ainsi, ce qui intéresse Guibal et Salaun dans cette question de fraude ou de falsification des élections comme l'appelle el-Aswany, c'est surtout le rôle des militants, des cybractivistes. Ceux-ci, à quelques jours des élections législatives, sont réunis dans une villa à Mohandessine. Ils sont «des militants des droits de l'homme, des opposants, des membres de la société civile » $(E T, 82)$ qui essaient de coordonner leurs efforts. Mais la réussite de

doi :10.12816/0004234 
l'affaire ne dépend pas d'eux, les électeurs ont un rôle non point médiocre, celui de renseigner cette fourmilière de militants, de la doter de preuves de la fraude par tous les moyens possibles. «Les gens doivent nous aider, dit Israa Abdel Fattah, nous envoyer par Twitter, par mails ce qu'ils voient, ils doivent être nos yeux » $(E T, 83)$. Toutefois, il faut dire que ce n'est pas par négligence, ni par paresse, ni par souci de jouer le rôle de leader que les membres de la fourmilière demandent aux autres de travailler, mais parce que la plupart des militants sont poursuivis par la Sécurité de l'Etat qui ne cesse de les harceler, de les arrêter toutes les fois qu'il y a une manifestation. Israa Abdel Fattah elle-même, militante qui fut à l'origine de la grève du 6 avril 2008, fut arrêtée plusieurs fois depuis lors. Bien que, après avoir été libérée, elle ait promis devant les caméras de «ne plus se mêler de politique »(83) la voilà qui recommence après deux ans, sans la moindre faille.

Cette jeunesse militante, dont Israa Abdel Fattah et beaucoup d'autres dont les noms figurent dans «l'Egypte de Tahrir», a en commun cette foi en Internet. On «sait le poids d'Internet»(ET,83) comme le dit Guibal et on reconnaît que c'est une arme redoutable qui peut parfaitement assener des coups qui puissent être mortels au Régime de Moubarak. Cette jeunesse qui excelle dans l'utilisation de la technologie, Guibal et Salaun la respecte doublement : une fois pour avoir été persévérante, une deuxième pour avoir été «progressiste » dans le sens de moderne, pour le fait d'appartenir à l'époque où ils vivent par opposition au Régime de Hosni Moubarak et ses partisans, qui, lui, vit dans le siècle passé.

De par sa confession musulmane, Alaa el-Aswany utilise ce sujet de la fraude des élections, pour rapporter cet acte à la religion et souligner ce que celle-ci pense des fraudeurs, dans le but bien sûr d'arrêter ces pratiques. Dans un premier temps, el-Aswany n'a aucun mal à réhabiliter le ministère de l'Intérieur (qui, aux yeux de tous, est le responsable qui conduit les élections) pour attaquer tout de suite «le président de la République qui prend la décision de frauder » (CRE, 79).

N'ayant rien trouvé dans les livres de jurisprudence islamique qui traite de la fraude électorale, el-Aswany recourt au syllogisme. Il assimile cet acte au faux témoignage car, selon lui, tous deux sont des «mensonges volontaires faits dans l'intention d'infirmer la vérité » 
(CRE,81). Or, les docteurs en jurisprudence sont tous d'accord sur le fait que le faux témoignage est l'un des péchés les plus graves ${ }^{14}$, contre lequel, le Coran, dans plusieurs versets, met vivement en garde ${ }^{15}$.» (CRE,81). L'auteur n'en reste pas là, il tient à montrer pourquoi le faux témoignage est un grand péché pour mieux souligner l'idée qui est à la base de la condamnation de l'acte, cette idée qui est également à la base même de la condamnation de la fraude. "Lorsqu'une personne fait devant un juge un témoignage contraire à la vérité, il commet une faute grave car, par son témoignage mensonger, il conduit à nier le droit $^{16}$ des uns pour le faire reconnaître d'une façon erronée aux autres. » (CRE, 81).Cette question de «droit» est d'une importance capitale, tout musulman sait qu'en ce qui concerne les droits des autres, Dieu ne pardonne à l'homme que si celui-ci obtient le pardon de celui dont il a usurpé le droit.

Arrivée à ce point, l'idée d'el-Aswany arrive à son terme et le reste est facilement compris. Le fonctionnaire qui participe à la fraude électorale fait un faux témoignage concernant des résultats mensongers. « Il empêche le candidat qui y a droit d'obtenir le poste qu'il mérite, tandis qu'il le donne à une personne qui ne le mérite pas ». (CRE, 81). Alaa el-Aswany n'en reste pas là. Il tient à souligner que « de (s)on point de vue, la fraude électorale est même bien pire que le faux témoignage, car ce dernier dépouille de son droit un individu et une famille, alors que la fraude électorale dépouille de son droit la nation tout entière. »(CRE,82). Qui veut commettre un pareil péché ? Et comment les fraudeurs ne sont-ils pas au courant du crime qu'ils commettent? D'après Alaa el-Aswany, les fraudeurs, «comme beaucoup d'Egyptiens » (CRE, 82) considèrent que «les élections démocratiques et l'alternance au pouvoir sont des questions secondaires qui n'ont pas de lien avec la religion ». (CRE,82) Le problème réside donc dans l'interprétation même de la religion, qui est pour beaucoup de musulmans une religion formelle où les rites sont totalement séparés de la bonne conduite avec les autres, idée primordiale chez Alaa el-Aswany qu'il reprend dans plusieurs de ses articles. Cette compréhension bornée

${ }^{14}$ Dans leur exécration du faux témoignage, certains docteurs sont allés jusqu'à le comparer à la non reconnaissance de l'unicité divine (al sherk billah).

${ }^{15}$ Alaa el-Aswany cite volontiers quelques versets du Coran pour appuyer son idée. Par exemple : «Ceux qui font des faux témoignages... » (Sourate de la Séparation du bien et du mal à partir du verset 72). Egalement : »Abstenez-vous de l'infamie, des idoles et abstenez-vous du mensonge » (Sourate du Pèlerinage à partir du verset 30)

${ }^{16} \mathrm{C}$ 'est nous qui soulignons.

doi :10.12816/0004234 
de la religion qui fait que le musulman sépare dans sa vie quotidienne les rites religieux qu'il pratique de la conduite qu'il a avec les autres prend ici une dimension nouvelle car cette compréhension faussée «nous prédispose au despotisme et nous fait plus docilement accepter l'oppression. » et l'auteur de conclure : «c'est ce qui explique que la dictature soit plus répandue dans les pays islamiques que dans les autres » (CRE, 82).

Ces deux étincelles de la révolution, à savoir l'affaire Khaled Said qui eut lieu en juin 2010 et les élections législatives frauduleuses qui se tinrent en novembre de la même année, Alaa el-Aswany n'en nie point l'importance en tant que causes directes de la révolution égyptienne du 25 janvier. Mais ce qui l'irrite le plus ${ }^{17}$, ce que lui considère comme la goutte qui a fait déborder le vase, comme la cause principale du soulèvement du peuple en ce début de 2011, c'est la préparation par l'Etat du fils cadet du rais à accéder au pouvoir et donc à succéder à son père. Comment Hosni Moubarak pouvait -il se permettre « après trente ans d'accaparation du pouvoir à coups de référendums truqués » (CRE, 9) de préparer l'accession au pouvoir de son fils Gamal ? Cette nomination, si elle eut lieu, aurait été une véritable insulte au peuple.

Déjà, il y a quelques années, traiter de ce sujet tabou était fatal. Il ne faut pas oublier le destin du sociologue égypto-américain Saad Eddine Ibrahim, ancien directeur de thèse de la Première dame, et militant des droits de l'homme, ce destin qui lui valut sept ans de prison rien que parce qu'il fut parmi les premiers qui ont osé approcher le sujet en parlant de 1'Egypte comme étant une gomlokeya ce que Guibal et Salaun traduisent par Répu-narchie. (ET,100).«Officiellement, pour avoir utilisé, sans permission, des fonds offerts par l'Union européenne aux $\mathrm{ONG}^{18}$ pour la supervision des élections. »(ET,101). Mais Alaa elAswany n'a pas peur. Il sait que le régime est arrivé à un point d'orgueil qu'il a désormais «la peau épaisse » comme les analystes devaient le qualifier plus tard après qui la révolution l'a reversé. El-Aswany sait que l'Etat, plus sûr que jamais de sa capacité de se protéger par la Sécurité de l'Etat (et peut-être même l'armée puisque Moubarak est un des leurs)

\footnotetext{
${ }^{17}$ Au moins d'après le nombre d'articles rassemblés dans l'ouvrage que nous étudions.

${ }^{18}$ Organisation non-gouvernementale.
}

609 
laisse dorénavant les écrivains militants « causer » afin qu'ils servent de soupape de sûreté contre le grondement du peuple. «En Egypte, dit l'auteur de l'Immeuble Yacoubian ${ }^{19}$, on a la liberté de bavarder. Le régime te dit c'est ça, cause, cause toujours. » (CRE,86).

Cette question de la succession de Gamal, ou de Jimmy, comme ses proches le surnomment, a commencé autour des années 1990. Elle se pose « avec instance » depuis le 26 juin 1995 (ET,97). Jour de l'attentat perpétré contre le président à Addis-Abeba par un commando islamiste. Ce n'est pas la première fois que le président échappe à un assassinat. Mais cette fois, « le fill de la Faucheuse est cependant passé très près. » $(E T, 97)$. En Egypte, beaucoup se demandent: qui dirigera le pays si Hosni Moubarak disparaît ?

Mais alors que Gamal venait d'entamer son ascension politique, on commençait à soupçonner que le président, sous l'impulsion de sa femme, réputée pour sa forte personnalite ${ }^{20}$, préparait son fils pour lui succéder.On commençait aussi à faire le lien entre le refus de celui-ci de désigner un vice-président, comme l'ont fait tous ses prédécesseurs et la « réservation » à Gamal du fauteuil présidentiel.

En novembre 2003, lors d'une allocution, le président eut un malaise devant les caméras qui transmettaient l'événement en directe : fausse alerte, le président grippé ne reprend le discours qu'après plus d'une demi-heure.

Gamal continua son ascension politique, utilisant bien sûr le PND comme instrument pour ce faire.Ce PND qui «fut toujours une des incarnations du pouvoir en Egypte. Sans lui, rien ne se faisait. Sans lui, rien ne pouvait exister. Depuis trente ans, il monopolise la vie politique. » (ET,60). Le parti tint en 2003 son congrès annuel. A grand frais, le fils du président invita des membres de partis politiques

${ }^{19}$ Citer ici L'Immeuble Yacoubian n'est pas dépourvu de sens. Dans ses romans, dont L'Immeuble Yacoubian, Alaa el-Aswany rassemble tous les maux dont souffre l'Egypte, ces maux qui ne sont que de véritables « ingrédients qui doivent mener à une catastrophe finale : l'injustice, la corruption, la fraude électorale, la répression policière, la torture, l'extrémisme islamiste, l'exil et, en arrière fond, lointain, le Grand-homme. » Nous pensons que le fait de causer et de causer encore sans que personne ne nous entende fut aussi, avant la révolution, un des grands maux de l'Egypte. D'où le besoin de citer ici le chef d'œuvre de l'auteur. CRE, p.5.

${ }^{20}$ On lui prête, plus qu'à son mari, d'être à l'origine de la mise sur orbite politique de leur fils Gamal. Plus qu'un fils à Papa, Gamal serait un fils à maman. En une quinzaine d'année, ce banquier, fondateur de Medinvest, un fonds d'investissement qui sera un instrument de son enrichissement, s'est transformé en un animal politique, grand ponte du parti au pouvoir et perçu, à l'étranger, comme un pair du régime. (ET, 88-89).

doi :10.12816/0004234 
américains et européens, ainsi que des personnalités internationales, à «suivre ses travaux ». (ET,99). «Opération marketing réussie pour Gamal Moubarak, soulignent Guibal et Salaun, bombardé héraut des réformes et du changement. » $(\mathrm{ET}, 99)$. Aidé par les meilleurs conseillers de son père ${ }^{21}$, et s'appuyant toujours sur le PND, Gamal en devint le chef en quelques années. Plus grave encore et plus significatif fut l'accueil que lui firent les pays du monde : il était reçu à l'étranger par les chefs d'Etat ou leurs conseillers. " C'est l'homme incontournable », commentent les auteurs français.(ET,99).Aussitôt, son emprise s'étendit: ses hommes entrèrent au gouvernement, aux postes clé de l'économie et bientôt, ce «cabinet Gamal » «dynamique, moderne, efficace, fut salué par les milieux d'affaires et les institutions financières internationales. » $(\mathrm{ET}, 100)$.

Bientôt des voix s'élevèrent. Kefaya est créé. Dans toutes les manifestations, on conspuait désormais «l'héritage du pouvoir qui était en train d'être élaboré. D'autant plus que« des casseroles, déjà nombreuses s'attachaient au nom de Moubarak $^{22} »(E T, 100)$. Mais le président, au lieu d'écouter son peuple, ne commenta point l'affaire. Quant à Gamal, il continuait à nier tout projet de transmission du pouvoir chaque fois qu'il était interviewé. Cependant, en 2007, le grandhomme modifia la Constitution.Les amendements constitutionnels verrouillèrent le système politique «par un habile mécanisme de signatures d'élus indispensables pour qui souhaiterait se présenter en indépendant. » (ET,31). Amendements destinés, semble-t-il à réserver le poste de président de la république au président et à ses fils et à réduire tout autre candidat à un simple figurant insignifiant dans la comédie de la transmission héréditaire du pouvoir, «rôle indigne qu'aucune personne qui se respecte ne peut accepter. » (CER, 63).

Entre temps, la communauté internationale continua à témoigner de son adhésion complète au projet de la succession et Jimmy fut reçu dans

\footnotetext{
${ }^{21}$ Ossama al-Baz et Zakkaria Azmi.

${ }^{22}$ «Le nom de Alaa, businessman florissant, a été plusieurs fois associé à des affaires de monopole et d'attribution délictueuses de marchés.(...) Mais, en 1997, quand le journal saoudien Al-Shark al-Awsat s'apprête à publier une enquête sur les finances des deux frères, promettant des révélations sur des commissions qu'ils auraient touchées lors de l'achat d'avions pour Egyptair, les fils présidentiels, préemptifs, attaquent en justice. Prison ferme pour les journalistes.
}

611 
les grandes capitales du monde : à Paris, à Washington. « Le blanc-seing est totale. » $(\mathrm{ET}, 101)$.

Comme nous venons de le voir, l'essentiel de l'histoire de l'ascension de Gamal est raconté par Claude Guibal et Tangi Salaun. Destiné au lecteur français, l'ouvrage, comme nous l'avons dit plus haut, présente une "radiographie » de l'Egypte au moment où celle-ci s'apprête à changer d'ère. Basé essentiellement sur des rencontres et des témoignages il ne fait que proposer une étude de l'état des lieux de la société égyptienne en ce moment. Les commentaires sont restreints, les analyses sont absentes. C'est au lecteur qu'incombe la tâche de tirer les conclusions qu'il veut.

L'approche de Alaa el-Aswany est totalement différente. Destiné au lecteur égyptien, arabe au moins, il suppose une certaine connaissance de l'actualité égyptienne qu'il se donne pour tâche, la plupart du temps, d'analyser et de commenter. C'est dans cette optique donc que ce sujet de l'ascension de Gamal est traité.

Pour Alaa el-Aswany, la question de la transmission héréditaire du pouvoir est l'étincelle de la révolution.Quoique l'élaboration de ce projet ait commencé très tôt, ce n'est qu'en ces quelques dernières années que cette transmission devient sujet d'actualité. Les Egyptiens réalisent que le projet est sur le point d'être exécuté. Alaa el-Aswany en donne les raisons sans détour. «A plus de quatre-vingt ans, le président Moubarak gouverne l'Egypte depuis trente ans et - avec tout mon respect - il ne peut pas, du fait de son âge et des lois de la nature, rester indéfiniment à son poste. » (CRE,21). L'inquiétude de l'auteur devint encore plus intense quand, il a vu Emad Adib, «journaliste émérite et proche de la présidence » intervenir dans un débat public avec « une déclaration unique en son genre »: il souhaitait -assurait-il - que «le président soit libéré de ses fonctions et il demandait que les présidents puissent se retirer en toute sécurité, c'est-à-dire sans que leur soient demandés des comptes, tant au plan politique que judiciaire, pour des faits accomplis pendant l'exercice du pouvoir ». (CRE, 21-22). Pour elAswany, il est peu imaginable qu'une personnalité publique telle que celle d'Emad Adib suggère une idée d'une telle importance et d'une telle précision sans avoir l'autorisation de la faire.

Ces signaux ajoutent à la confusion de la scène politique. Nous ne savons pas si le président va se retirer ou s'il va rester à son poste. Ce qui est encore pire, c'est qu'un jour il se peut qu'on n'ait même pas le choix «Moubarak peut se trouver à n'importe quel moment obligé de prendre sa retraite. $\gg(C E R, 65)$. Toutefois, quelque puisse être l'importance de cette question de la retraite du père ou de l'avènement

doi :10.12816/0004234 
du fils, derrière les écrits de l'auteur transparait un souci non moins grand.

Ce souci, c'est de voir toute la politique de l'Egypte tourner dans l'orbite de l'héritage du pouvoir. Comment un pays tel que le nôtre peut-il se trouver contraint de sacrifier son passé récent, son présent et son futur rien que pour permettre à un fils à papa ou à maman d'hériter le pouvoir de son père ? Guibal et Salaun ont souligné à plusieurs reprises l'adhésion internationale au projet des Moubarak mais sans montrer les raisons de cette adhésion. Ils ont relaté les faits qui prouvent l'existence de l'idée d'héritage du pouvoir dans l'esprit du président (et quelle audace de vouloir réaliser une idée pareille dans une république) mais ils n'ont pas précisé si Gamal, en tant que personne, méritait vraiment d'être président de l'Egypte que «finalement, son nom de famille est son principal handicap », comme l'a dit un jour un diplomate européen $^{23}$ (ET, 99).

C'est à ces deux points essentiels à savoir les raisons de l'adhésion internationale et les raisons du refus de Gamal président, que l'écrivain égyptien consacrera la plus grande partie de son travail en ce qui concerne le sujet de la succession. En ce qui concerne le premier point, Alaa el-Aswany accuse le régime de «s'évertuer» à préparer l'accession de Gamal au pouvoir à l'intérieur et à l'extérieur. A l'intérieur à travers deux plans exécutés simultanément. Le premier consiste à créer « une officine ad hoc en charge de la transmission héréditaire ",, composée de journalistes, d'hommes politiques, de responsables des médias ainsi que de professeurs de droit, dont l'unique mission est de «préparer le peuple égyptien à en accepter l'idée » (CRE, 28,29). Des dizaines de rencontres ont été organisées, de nombreuses visites ont été planifiées à son intention dans les villages et les quartiers populaires «au cours desquelles quelques familles de miséreux ont été choisies, en coordination avec les services de sécurité, pour être photographiées en train de serrer sa main et de chanter ses louanges » (CRE,24). Ceci évidemment se fait séparément du travail du PND dans le même but. Quant au deuxième plan, il consiste à se débarrasser

${ }^{23}$ C'était lors de « l'opération marketing », le congrès annuel du PND tenu en septembre 2004 où un très grand nombre de personnalités internationales ont été invités.

613 
physiquement ou moralement de toute figure égyptienne susceptible d'attirer l'admiration des Egyptiens ou faisant preuve d'une certaine popularité ou alors montrant une certaine compétence à rassembler les masses. Les exemples ne sont pas rares. Nous nous rappelons l'accueil que l'Etat a réservé au docteur Ahmed Zoeil et le projet de son université avant qu'il n'ait joui du rassemblement du peuple autour de lui et avant que celui-ci ne lui demande de présenter sa candidature aux élections présidentielles ${ }^{24}$; même sort réservé au docteur Mohamed elBaradei. Nous nous rappelons du prédicateur Amr Khaled, de la popularité inouïe dont il jouissait avant de se trouver contraint de quitter son pays. Le docteur Mamdouh Hamza est peut-être le seul à avoir pu échapper à la persécution de 1'Etat parce que son histoire n'a pas été très connue semble-t-il ${ }^{25}$.

Cependant, les efforts du régime n'ont pas été limités à l'intérieur du pays, mais ont également été déployés à l'étranger, au point que «le principal objectif de la politique extérieure égyptienne est devenu d'acquérir le soutien des pays occidentaux à Gamal Moubarak - les intérêts de l'Egypte, ses richesses et son honneur étant le prix à payer pour leur approbation. »(CRE,22). Les exemples abondent dans les deux ouvrages étudiés. A titre d'exemple, « sachant que la clef du cœur de l'Occident se trouve entre les mains d'Israël », (CRE,22) et qu'exécuter les désirs de (celle-ci) était le moyen assuré de satisfaire l'Amérique », (CRE,51) jamais le régime de Moubarak n'a osé attaquer la politique israélienne par peur de susciter le mécontentement des Etats-Unis. Pour

${ }^{24}$ Quelques mois plus tard, le président Obama s'empressa d'en faire son conseiller scientifique pour mettre ses grandes connaissances au profit du développement des Etats-Unis.

${ }^{25}$ Le docteur Mamdouh Hamza est l'un de nos plus grands professeurs d'ingénierie. Il a supervisé l'exécution de dizaines de grands projets en Egypte et dans différents autres pays du monde, parmi lesquels les Etats-Unis, la Grande-Bretagne et le Japon. Il a obtenu d'importants prix internationaux. Connu pour son patriotisme et son sens du devoir, il a déclaré, après les inondations qui ravagèrent la province d'Assouan il y a quelques années qu'il était prêt à bâtir bénévolement des maisons pour les indigents qui avaient été chassés des leurs. Les dons qui affluèrent immédiatement atteignirent vingt-huit millions de livres. Il alla à Assouan pour suivre lui-même à ses frais, les travaux de construction. Le gouverneur l'accueillit chaleureusement et lui accorda un terrain pour la réalisation du projet. En trois semaines, il construisit vingt-neuf unités dont il réduisit le coût au chiffre sans précédent de trente-cinq mille livres. Au lieu de lui demander d'étendre son projet à toutes les provinces de l'Egypte, «le régime égyptien s'est retourné contre lui ». Peut-être, entre autres raisons, allait-il jouir d'une grande popularité surtout qu'il allait nuire aux grands : les logements réalisés par le gouvernement revenant à quatre-vingt mille livres.

doi :10.12816/0004234 
ne pas mettre également en péril sa popularité en Egypte et dans le monde arabe « où elle est au plus bas » (CRE,49), Moubarak entame alors «un ballet étonnant» faits d'entrechats et d'éloignements, sans jamais atteindre la rupture. "Il ne se rend (à Israël) qu'une fois, en 1995, sommé par la Maison-Blanche d'assister aux funérailles de Yitzhak Rabin. » (ET,94).

Par les services rendus à Israël dans le but d'assurer la transmission du pouvoir, le régime égyptien «a dépassé ses propres performances » (CRE,22) assure Alaa el-Aswany. A le croire, « entre l'année 2005 et aujourd'hui $^{26}$, Israël a reçu de l'Egypte tout ce qu'il n'avait pas pu obtenir depuis les accords de Camp David »(CRE,24-25): retour de l'ambassadeur égyptien, contrats de fourniture de gaz, de pétrole et de ciment, et, mieux encore, tentative de convaincre ou de contraindre les Palestiniens à se plier à toutes les exigences de l'Etat hébreu. L'affaire alla même jusqu'à la fermeture du terminal de Rafah, comme contribution au siège des Palestiniens et au châtiment du mouvement Hamas jusqu'à ce que ce dernier se plie à la volonté israélienne.

Il faut dire qu'en échange de ces services, le soutien international implicite a été accordé au régime de Moubarak, les présidents occidentaux ne cessent de le glorifier en toute occasion, de le remercier de temps à autre pour ce qu'ils appellent « ses efforts en faveur de la paix » (CRE,23) le président Obama allant jusqu'à le considérer comme «un chef sage avançant dans le sens de la démocratie »(CRE,23). Curieux. Les Etats-Unis savent que le président égyptien truque les élections, opprime ses opposants, pratique la torture et sape tous les jours les règles de la démocratie. En Iran, (le premier ennemi d'Israël), la moindre fraude électorale entraîne «immédiatement» une mobilisation politique en Occident en faveur de la démocratie.(CRE, 23). Emadeddine Hussein, un éditorialiste du journal indépendant $A l$ Chorouq al-Gedid partage le même avis : «Croire, dit-il, que les EtatsUnis et l'Union européenne s'intéressent au sort des législatives égyptiennes relève de la sottise. Washington, Bruxelles ou Tel Aviv seront-ils satisfaits si ce sont les Frères musulmans qui remportent des élections organisées dans un climat intègre? La réponse est non. L'Occident souhaite bien sûr, dans l'idéal, voir les pays qu'il soutient

${ }^{26}$ Quelques mois avant la révolution.

615 
jouir de la démocratie et du multipartisme. Mais pour réserver ses intérêts, il a toujours soutenu des régimes oppresseurs et il continuera à le faire. » $(\mathrm{ET}, 101)$.

Autre exemple : des militants étrangers appartenant à de nombreux pays à travers le monde sont venus en Egypte pour proclamer leur totale solidarité avec les Palestiniens assiégés à Gaza et leur faire parvenir de la nourriture et des médicaments. Pour les dissuader d'accomplir leur mission, les autorités égyptiennes leur ont offert des visites touristiques gratuites. Refusant de laisser tomber la responsabilité qu'il s'était donnée, «la police égyptienne a alors donné l'assaut contre leur rassemblement, en jetant au sol les manifestants et en les frappant sauvagement $\gg$ (CRE,199-200).

Dernier exemple : le gouvernement égyptien a reconnu qu'il était en train de construire un mur d'acier sous la terre, tout au long de la frontière avec Gaza de façon à murer les tunnels utilisés par les Palestiniens pour contrecarrer le siège qu'il leur est imposé par Israël et ceci sous différents prétextes qui, selon el-Aswany, «ne pourraient même pas convaincre un petit enfant $»^{27}(\mathrm{CRE}, 49)$. A croire l'auteur, le mur est bâti sous financement et contrôle américain. Cette construction du mur d'acier va de pair avec la fermeture du terminal de Rafah et l'interdiction faite aux convois de secours arabes et internationaux à entrer à Gaza. A son tour, peu de temps après avoir pris ces mesures contre les Palestiniens, Margaret Scobey, l'ambassadrice américaine au Caire a déclaré : «Je crois que le niveau de démocratie en Egypte est satisfaisant. »(CRE,51).

A l'instar de l'auteur de L'Immeuble Yacoubian, nous ne devons pas nous étonner ni de l'attitude du régime de Moubarak d'une part ni de celui des Etats occidentaux et des Etats-Unis d'autre part. Pour ce qui est du deuxième point, comme le dit savamment El-Aswany, la dualité des critères a toujours été une caractéristique des gouvernements

${ }^{27}$ Le régime égyptien se dit libre de construire ce qu'il veut sur ses frontières (il fait semblant d'oublier le droit international qui l'oblige à respecter le droit des autres), il dit que les tunnels sont employés illégalement - pour faire entrer des armes pour les terroristes (feignant de ne pas se rendre compte que ces armes peuvent également passer par la Libye et le Soudan). Il soutient l'idée que la sécurité nationale est notre ligne rouge (faisant semblant que notre véritable ennemi est Israel ce qui veut dire qu'il est dans notre intérêt national de soutenir les Palestiniens). Enfin les responsables égyptiens disent qu'ils craignent une émigration massive de Palestiniens en Egypte, (ceci est ridicule car les Palestiniens ne viennent que pour acheter ce dont ils ont besoin et puis retournent chez eux.) (CRE, 47-51).

doi : 10.12816/0004234 
occidentaux. (CRE,23). Pour ce qui est de Moubarak, il est clair que rien ne l'aurait obligé de se comporter de la sorte que son besoin du soutien de l'Occident et surtout de l'Amérique pour son projet de transmission héréditaire du pouvoir. El-Aswany voit là «un exemple grave des conséquences du régime dictatorial »(CER,52). Car seuls les régimes démocratiques travaillent pour l'intérêt de leurs peuples, parce que leurs responsables élus, doivent leurs postes au peuple. Une dernière constatation: le régime de Moubarak fut depuis longtemps en contradiction avec l'intérêt du peuple égyptien. Le régime, en agissant de la sorte, ne mit pas seulement en péril sa popularité en Egypte et dans le monde arabe mais «souilla» également sa réputation internationale (CRE,49): la «complicité entre le gouvernement égyptien et Israël dans le siège de Gaza » étant une expression qui revient sans cesse dans la presse internationale. » (CRE, 202).

Cependant, une question se pose: dans quelle mesure Gamal Moubarak méritait les sacrifices de l'Egypte et n'était-il pas, en toute objectivité, le mieux placé pour devenir président de la république ?

En premier lieu, le refus de Gamal président est, pour Alaa elAswany, une question de principe. Dans des termes plus populaires que ceux de Saad Eddine Ibrahim, l'auteur refuse comme la majorité des Egyptiens, que l'Egypte républicaine devienne pratiquement une monarchie « où un dauphin est appelé à hériter du trône occupé par son père » (CRE,24).Ceci relève d'une injustice flagrante car ce grand pays «n'est ni une ferme, ni un élevage de volailles appartenant à une personne, quelle qu'elle soit et quelle que soit sa position. ${ }^{28} \gg(\mathrm{CRE}$, 28). Déjà, rappellent Guibal et Salaun, quand Bachar el-Assad succède à son père, Hafez, à la tête de la Syrie en 1999, «1'Egypte entière se gaussa ouvertement de cette république dynastique » $(\mathrm{ET}, 100)$. Et quand, plus tard le rais semble prendre des mesures pour transmettre le pouvoir à son fils, «1'Egypte entière s'émeut de ce scandaleux passage en force »(CRE,101). «L'Empereur, sa femme et les petits princes. Plus encore que le rais, c'est la famille qui est détestée par la population » $(E T, 102)$. Gamal, précisément, n'a jamais été proche du

\footnotetext{
${ }^{28}$ A la page 247 du même ouvrage, (mais dans un autre article), el-Aswany compare l'Egypte réduite au statut de monarchie à « un élevage de poulets qu'on lègue par testament. »
} 
peuple, «à 47 ans, il reste toujours distant » (ET,102). Alaa el-Aswany ajoute que, Gamal Moubarak est peut-être un grand expert de la banque et des affaires, mais il n'a aucune sorte de don ou d'expérience dans le domaine politique. (CRE, 24). Malgré les grands efforts déployés donc par le régime pour lancer Gamal Moubarak, ce dernier a totalement échoué à convaincre la plupart des Egyptiens de son « aptitude au poste de président »(CRE,65).

Cependant, il faut souligner que, comme nous l'avons dit plus haut, ce ne sont pas tous les Egyptiens qui étaient contre la transmission héréditaire du pouvoir. Lors des dernières élections présidentielles avant la révolution, le Pape Chnouda III a demandé aux coptes d'aller voter pour le président. De même, sa Sainteté a proclamé son soutien catégorique à Gamal Moubarak ce qui voulait dire que «l'Eglise égyptienne accueillait favorablement la transmission héréditaire du pouvoir » du père au fils (CRE,156). Entre autres les raisons qui, selon el-Aswany aurait dû empêcher le Pape de se prononcer en faveur de Gamal, c'était le fait qu'il représente une autorité spirituelle et non politique et comme les Egyptiens se battent pour l'instauration d'un Etat séculier en Egypte où tous les citoyens seraient égaux, cela implique une séparation entre la religion et la politique (CRE,161). De même, sa Sainteté est un égyptien qui vit en Egypte et qui est au courant de tous les maux dont souffrent les Egyptiens, « est-il conforme donc à l'enseignement du Messie de soutenir un régime corrompu et oppressif, demande l'auteur ? »(CRE,157) Cependant, el-Aswany sait que le Pape «craint pour les coptes que les élections démocratiques soient susceptibles d'amener au pouvoir les Frères musulmans » (CRE,158). La vérité est que «le rôle et l'influence des Frères musulmans ont été volontairement exagérés par le régime » $(\mathrm{CRE}, 158)$ pour que celui-ci continue à accaparer le pouvoir et réduire au silence toute tentative de changement $^{29}$. Le nombre et l'influence des Frères musulmans ne sont pas tels qu'ils puissent remporter la majorité à des élections honnêtes auxquelles le peuple participerait. Quant aux victoires remportées par les frères au cours de ces dernières années, el-Aswany souligne qu'elles n'ont pas eu lieu grâce à leur popularité mais parce que «les gens se sont détournés des élections. »(CRE,158).Et el-Aswany de

${ }^{29}$ El-Aswany rappelle que les Frères, alors qu'ils se trouvaient au sommet de leur force, ne sont pas parvenus à obtenir un seul siège au parlement élu en 1950, au cours des dernières élections libres avant la révolution. Ce fut cette fois-là encore un raz de marée du Wafd qui donna la majorité à ce parti.

doi :10.12816/0004234 
conclure : «ce sont des élections propres qui peuvent écarter le danger de l'extrémisme religieux et non le contraire. » (CRE,159).

Nous voyons bien qu'Alaa el-Aswany s'acharne à défendre la cause du droit de l'Egypte, premier pays qui ait connu la démocratie en Proche-Orient, à choisir elle-même son président à travers des élections libres et intègres. Ce n'est pas tout, el-Aswany dans sa bataille réfutera un à un tous les arguments en faveur de Gamal.

Les promoteurs de Gamal ne disposent que de trois arguments «fallacieux » sur lesquels ils reviennent sans cesse (CRE, 29):

Premièrement : ils disent que Gamal Moubarak est un jeune homme poli et très instruit auquel il n'y a pour le moment pas d'alternative d'autant plus qu'il sera le premier président civil de l'Egypte depuis la révolution, ce qui sera un pas vers la démocratie. Pourquoi donc ne l'accepterons pas, à condition qu'il s'engage à ne faire que deux mandats présidentiels ? Selon el-Aswany, hormis le cas de Gamal, le niveau d'éducation et d'instruction ne peut pas être à lui seul, l'unique critère dans le choix du président surtout qu'en Egypte, il y a «des centaines de milliers de personnes polies, qui ont obtenu des diplômes de l'enseignement supérieur et qui ont un excellent niveau en anglais et en français. Sont-ils tous aptes à la présidence ?» se demande l'auteur. Que Gamal soit pour le moment la seule alternative ceci n'est pas vrai. Les Egyptiens, se trouvant plus poussés chaque jour plus que l'autre à accepter Gamal ont commencé à penser à d'autres personnes, plus compétentes à assumer cette responsabilité comme le docteur Mohamed El Baradei, le ministre Amr Moussa ou le docteur Ahmed Zoueil ${ }^{30}$ « Il y a en Egypte assez de compétences et de cerveaux pour répondre aux besoins de dix pays. » (CER,30). Quant à l'allégation selon laquelle Gamal Moubarak serait un président civil, elle est également fallacieuse car « ce qui définit la nature du régime, ce n'est pas le métier du président, mais la façon dont il a obtenu le pouvoir »(CRE,30). Pour illustrer son idée, Alaa el-Aswany donne l'exemple de Bachar el-Assad. Celui-ci, bien qu'il soit une personnalité civile, est placé par une institution militaire despotique. Le régime est donc de nature despotique. En revanche, il y a dans les pays démocratiques des

\footnotetext{
${ }^{30}$ Ces noms proposés par Alaa el-Aswany sont les noms qui sont à l'esprit du peuple avant la révolution.
}

619 
militaires qui ont quitté leurs postes dans l'armée pour se présenter à des élections libres comme par exemple Charles de Gaulle ou Colin Powel. Selon el-Aswany, si Gamal Moubarak accède à la présidence de l'Egypte, cela ne mettra pas fin au régime militaire existant mais « cela apportera une calamité supplémentaire : nous aurons simultanément le despotisme et la transmission héréditaire du pouvoir. »(CRE,30). Ceux qui prétendent que Gamal va se contenter de deux mandats présidentiels, el-Aswany leur répond que l'exemple de son père est toujours présent dans les esprits. Hosni Moubarak avait promis un jour qu'il ne restera au pouvoir que pour deux mandats. Sans des réformes politiques sérieuses, rien ne contraindra Gamal à ne pas suivre l'exemple de son père.

Deuxièmement : Les supporters de Gamal disent que les Egyptiens ne sont pas préoccupés ${ }^{31}$ par la question de la démocratie, et que par ailleurs, ils ne sont pas qualifiés ${ }^{32}$ pour vivre dans une atmosphère démocratique c'est-à-dire qu'ils n'ont pas les qualités nécessaires pour la pratiquer, compte tenu de l'analphabétisme ${ }^{33}$. Ils affirment que s'il y avait des élections libres ;les Frères musulmans les emporteraient et s'empareraient du pouvoir. Alaa el-Aswany refuse catégoriquement ces prétentions.

Pour ce qui est du premier point, celui du désintérêt des Egyptiens de la démocratie, l'auteur pense que le fait même d'organiser des manifestations et de faire des grèves dans l'atmosphère d'oppression qui régnait sous Moubarak, même si les participants sont peu nombreux, est déjà une preuve d'intérêt à la démocratie. Il rappelle qu' «avant l'apparition d'El Baradei, avaient été créés de nombreux mouvements nationaux pour le changement dont le plus important était le mouvement Kifaya $^{34}$ auquel revient le très grand mérite d'avoir brisé, chez les Egyptiens, le mur de la peur. » (CRE,66). Il faut souligner le fait que le mécontentement de ce mouvement né en 2004 à l'écart de des partis de l'opposition officielle, s'était cristallisé très tôt sur le rejet d'une " dynastie Moubarak ${ }^{35} »($ ET,96).Le commentaire des auteurs français

\footnotetext{
${ }^{31}$ Nous soulignons.

${ }^{32}$ Nous soulignons.

${ }^{33}$ Nous soulignons.

34 Premier mouvement contestataire à avoir demandé, en 2004, le départ du président Moubarak.

${ }^{35}$ Déjà, le 12 décembre 2004, à peine une centaine rassemblée sur un bord de trottoir devant la Haute Cour de justice crie « Hosni Moubarak, Kefaya! Gamal Moubarak, Kefaya ! Suzanne Moubarak, Kefaya !» (ET, 96).
} 
témoigne de l'ampleur de l'événement. « Là où, à Paris ou à New York, un tel événement n'attirerait pas l'attention du moindre journaliste, pour l'Egypte en ces années-là, il est de taille.Car c'est la première fois qu'on entend critiquer ainsi le président et sa famille. Quand même... il faut oser. » $(\mathrm{ET}, 96)$. Il ne fallait que quelques mois pour les activistes de Kefaya, pour devenir «les trouble-fête du paysage politique égyptien » et pour conspuer désormais dans toutes les manifestations, « l'héritage du pouvoir » qui était en train de se mettre en place. (ET,96), (ET,101). Quelques années plus tard, l'Egypte est « submergée » par les grèves et les mouvements de protestation, « comme cela n'avait pas eu lieu depuis la révolution de 1952 »Cette contestation sociale généralisée, avance elAswany, annonce un changement inévitable et elle n'est pas du tout étrangère à la démocratie. Les mouvements de protestations continus expriment la revendication par le peuple égyptien d'une justice qui ne peut advenir que grâce à des réformes démocratiques.

Pour ce qui est du second point, que les souteneurs de Gamal Moubarak soulèvent et dans lequel ils prétendent que les Egyptiens, même s'ils aspirent à vivre dans la démocratie ne sont pas qualifiés pour la pratiquer, Alaa el-Aswany ne peut que s'indigner, autant de «"l'indécence» de l'argument que de «l'ignorance fautive de l'histoire » de ceux qui en discutent. Il suffit de prendre un livre d'histoire moderne de l'Egypte pour savoir que l'expérience démocratique de notre pays a commencé avant celle de nombreux pays européens, en 1866, lorsque le khédive Ismail fonda la première chambre de députés qui, au début, était consultative, mais dont, rapidement, les membres se battirent pour arracher de véritables compétences. De 1882 à 1952, les Egyptiens ont lutté et sacrifié des milliers de martyrs pour deux objectifs: l'indépendance et la constitution. «La libération de l'Egypte de l'occupation britannique a toujours été liée dans la conscience des Egyptiens à l'instauration de la démocratie. »(CRE,31). De même, le sens de la démocratie est l'égalité, la justice et la liberté et il n'est pas possible qu'un peuple les mérite plutôt qu'un autre. (CRE,31).

Pour ce qui est du troisième point qui lie la pratique de la démocratie à l'alphabétisme, Alaa el-Aswany est clair. L'analphabétisme n'interdit pas la démocratie et nous avons dans l'expérience de l'Inde un exemple flagrant. Ce pays dont le niveau d'analphabétisme est élevé, est parvenu « à bâtir démocratiquement un grand pays en peu d'année »(CRE,31). 
De même, le niveau d'analphabétisme avant la révolution n'a pas empêché le parti Wafd de remporter d'écrasantes victoires toutes les fois que des élections honnêtes étaient organisées. «Les paysans égyptiens analphabètes ont toujours voté pour le wafd contre les propriétaires terriens du parti libéral constitutionnel ».(CRE,32). Selon l'auteur égyptien, les gens n'ont pas besoin d'avoir un doctorat en droit pour savoir si le pouvoir dans leur pays est oppressif et corrompu ou non. Au contraire, le sentiment des gens simples est souvent plus proche de la vérité que les théories des intellectuels et leurs interminables discussions. Alaa el-Aswany explique en disant que « les intellectuels et les politiciens analysent tout à partir de théories et d'idées préconçues. Ils parlent beaucoup, se lancent dans des controverses inextricables et ne tombent jamais d'accord ;tandis que les gens ordinaires, même s'ils sont moins instruits, jouissent souvent d'un instict politique sain qui leur permet de se faire avec sagacité une opinion perspicace et d'adopter avec une incroyable simplicité la position juste. ${ }^{36} \gg$ (CRE, 109).

Ce sentiment des gens simples, cette boussole infaillible qui les guide dans leur choix, (ici politique), el-Aswany l'appelle « la conscience politique aigue ${ }^{37} »$. Il pense qu'elle est innée, et qu'elle a toujours fait partie de la composition de la personnalité égyptienne ${ }^{38}$. «A chaque

${ }^{36}$ Quarante ans après le décès de Gamal Abd el Nasser, nous continuons à discuter de ses fautes et de ses réalisations, mais le peuple égyptien s'est exprimé au moment de sa mort, lorsque des millions de personnes sont descendues dans la rue pour l'accompagner à sa dernière demeure. Ces gens simples qui fondaient en larmes de tristesse, comme des enfants, avaient tout à fait conscience de ses fautes. Ils savaient que leur chef défunt était à l'origine d'une cruelle défaite de l'Egypte et de la nation arabe, mais ils se rendaient compte également que c'était un grand dirigeant, d'une fidélité rare à ses principes et qui avait mis tous ses efforts et toute sa vie au service de la nation.

${ }^{37}$ Cette idée, Alaa el-Aswany la développe longuement dans un autre article qui fait toujours partie du même ouvrage.

${ }^{38}$ Pour prouver son point de vue, Alaa el-Aswany raconte une histoire anecdotique. «Lorsque, en 1919, les Egyptiens se sont soulevés contre l'occupation britannique, Saad Zaghloul se rendit à Paris pour exposer les revendications de la nation égyptienne devant la conférence de paix réunie à la suite de la première guerre mondiale. Le gouvernement anglais manœuvra alors habilement en envoyant en Egypte une commission d'enquête présidée par Lord Milner, le ministre des colonies. Les Egyptiens se rendirent immédiatement compte qu'il s'agissait d'un stratagème. Ils comprirent que toute coopération avec la commission Milner détruirait la crédibilité de Saad Zaghloul en tant que dirigeant mandaté par le peuple égyptien. A son arrivée au Caire, la commission Milner fut accueillie par un boycott total. Aucun homme politique égyptien n'accepta de coopérer avec elle, à

doi :10.12816/0004234 
page, sans exception, de l'histoire de l'Egypte nous retrouvons, chez le peuple égyptien, cette conscience politique aigue. »(CRE,109).Il ne partage donc pas l'avis de nombreux intellectuels qui pensent, comme l'ancien patron de presse Hicham Kassem, éternel militant de la société civile que : «l'Egypte n'est pas la Tunisie, la conscience politique y est moins développée. ${ }^{39}$ »(ET, 15).

Et puis, pour continuer le troisième point, Alaa el-Aswany pense que de toute façon, il y a en Egypte, quarante millions de gens instruits et ce chiffre, pense-t-il, est largement suffisant pour garantir la réussite de l'expérience démocratique.

A ceux qui prétendent qu'en cas d'élections libres les Frères musulmans s'empareraient du pouvoir, Alaa el-Aswany assure que « la taille et l'importance du mouvement des Frères musulmans ne sont pas telles qu'ils puissent constituer une menace contre le régime égyptien, qui agite toujours cette idée pour justifier la dictature ${ }^{40}$. (CRE,51) Cependant, même à supposer que les Frères musulmans remportent un jour une victoire électorale possible, ceci serait le choix des Egyptiens qu'il faudrait respecter autant que les premiers respecteraient les règles démocratiques, les Frères musulmans étant des citoyens égyptiens qui ont le droit de remporter des élections et de participer au pouvoir. (CRE,32). Seule la réforme démocratique et l'alternance du pouvoir sont capables de mettre fin à l'extrémisme religieux. La dictature, elle, peut toujours réprimer et écraser les mouvements extrémistes mais jamais elle ne peut lui mettre un terme. «Des germes d'extrémisme resteront toujours cachés sous la surface attendant la première occasion pour ressurgir. » (CRE,32).

Enfin, aux propagandistes qui s'interrogent sur le droit de Gamal Moubarak de présenter sa candidature à la présidence de la république

tel point que Mohamed Saad Pacha, alors président du Conseil des ministres, démissionna de ses fonctions pour ne pas être contraint de traiter avec le responsable britannique. On raconte qu'un jour Lord Milner s'était perdu dans les rues du Caire. Son chauffeur avait voulu demander son chemin à un passant et celui-ci lui avait répondu : 'Dis à ton patron de le demander à Saad Pacha à Paris'. Le résultat de cette unanimité fut l'échec de la commission Milner. Le gouvernement britannique fut obligé de se soumettre à la volonté égyptienne et de négocier directement avec Saad Zaghloul.

${ }^{39}$ Témoignage pris le lendemain de la révolution, le mercredi 26 janvier 2011.

${ }^{40}$ Pour plus de détails sur ce point, Cf. Supra, p.15.

623 
comme n'importe quel citoyen égyptien, el-Aswany insiste qu'il ne lui nie aucun droit. Mais, selon lui, ceci suppose d'abord de bâtir un régime démocratique donnant l'égalité des chances à tous les candidats, de réviser la Constitution de manière à permettre une concurrence loyale, d'organiser enfin des élections honnêtes contrôlées par des juges indépendants et que ces élections se déroulent dans une atmosphère saine et détendue sans interférence de la police ou des hommes de main et sans fraude. C'est alors seulement que Gamal Moubarak pourra présenter sa candidature à la présidence de la république.

Et encore ! Même dans des conditions démocratiques, les chances de Gamal Moubarak à devenir président de la république auraient été minimes. Pourquoi ? Parce que simplement il s'appelle Gamal Moubarak. Et, en ce 25 janvier 2011, les jeunes sans illusions, les cyberactivistes qui sont descendus dans la rue criaient leur refus « du système sécuritaire en place en Egypte ${ }^{41}$ (et) leur ras-le-bol d'un pays dirigé depuis bientôt trente ans par le même homme, Hosni Moubarak, 82 ans... » $(\mathrm{ET}, 12)$.

«Trente ans de Moubarak, ça suffit. Tout le monde est fatigué » (ET, 16). Oui. Trente ans au pouvoir c'est beaucoup dans une monarchie, ce n'est pas acceptable dans une république. On a droit de parler d'un président qui «s'accroche ${ }^{42}$ au pouvoir jusqu'à son dernier souffle », (CRE, 54). Mais cette phrase, el-Aswany l'a écrite avant la révolution. Et si l'on attendait les dix-huit jours de la révolution? «Le 10 février 2011, alors que le crépuscule tombe sur le Caire, retranché dans son Palais présidentiel d'Orouba, dans le quartier d'Héliopolis, Hosni Moubarak s'agrippe encore au pouvoir. » (ET, 85).

D'après le témoignage des auteurs de «l'Egypte de Tahrir » le mandat d'Hosni Moubarak devenait, depuis presque dix ans, intolérable aux Egyptiens. Comment donc supporter son fils ? Sans le dire d'une manière explicite, le livre s'ouvre sur une phrase tout à fait significative et pleine d'insinuation. Après avoir indiqué la date du jour, 25 janvier $2011^{43}$, les auteurs écrivent: «C'est une aube paresseuse, un matin aux airs faussement tranquilles, où l'Egypte dort, flambée par la fatigue.. »

\footnotetext{
${ }^{41}$ Puisque c'est le jour de la fête de la police.

${ }^{42}$ Pour décrire la relation du président avec le pouvoir el-Aswany utilise généralement le verbe « s'accrocher» (ex. pages. 9, 54, 62, 157) Le verbe «accaparer» le remplace parfois (ex.p. 9).

${ }^{43}$ C. Guibal et T. Salaun ont choisi de commencer leur livre par la description chronologique, jour par jour, de la révolution et de faire ensuite une sorte de flash-back pour décrire la vie de l'Egypte en cette dernière décennie.
}

doi :10.12816/0004234 
(ET, 11). Etant donné que c'est un jour ferrié (la fête de la police), le lecteur croit, dans un premier temps, que c'est là la cause de la paresse, de la grasse matinée que font les Egyptiens en ce jour, mais les auteurs le harcèlent «... flambée par la fatigue d'une vie éteinte, nourrie de frustration, d'impuissance, d'ennui »(ET, 11). L'Egypte devenait à l'image de son vieux président, elle devient paresseuse, elle dort, elle est fatiguée à cause de la vie éteinte qu'elle mène bon gré mal gré.

La génération qui souffre le plus de cette atmosphère lugubre et austère et dont el-Aswany se fait le porte-parole, c'est la génération des années quatre-vingt. Pendant la révolution, tous répètent les mêmes mots : «Pas de liberté politique » $(\mathrm{ET}, 14)$; «Les gens n'ont pas accès à leurs droits. Je n'ai pas choisi Moubarak. Personne ne l'a choisi, il a été désigné » $(\mathrm{ET}, 16)$. «Il était là avant ma naissance ! Je ne l'ai pas choisi ! Personne ne 1'a choisi d'ailleurs, il a été désigné. Alors qu'il se casse maintenant ! (ET, 86). C'est vrai. En 1981, Anouar el-Sadate a été assassiné par un commando islamiste. Son vice-président, l'ancien commandant des forces aériennes du pays, un militant discret, a pris la suite. Il s'appelle Hosni Moubarak. A croire Guibal et Salaun, cette génération âgé de 30 ans et qui, par conséquent, n'a connu d'autres présidents que lui, forment «les deux-tiers de la population égyptienne. » $(\mathrm{ET}, 16)$.

Un autre jeune prie pour obtenir un visa, partir ailleurs, trouver un job. Il râle lui aussi contre le pays, le système bien qu'il appartienne à la riche bourgeoisie. «Je veux vivre une autre vie, dit-il, les yeux dans le vague. Je veux avoir des envies, sentir que les choses sont possibles. Ici, les gens ont peur de bouger, même quand ils ne risquent rien à le faire. (...) Je veux que ça change, ici. Je veux quelque chose de nouveau. » (ET, 59).

Stabilité. Oui. C'est là peut-être, en effet, « la prouesse principale de Hosni Moubarak» (ET, 91). Mais nous pouvons parler aussi d'immobilisme. En fait, depuis qu'il a hérité, à la mort de Sadate du «plus grand Etat du monde arabe » et en vingt-neuf ans de règne - le plus long qu'ait connu l'Egypte depuis le sultan Mohammed Ali -, Hosni Moubarak a toujours fait de la prudence le moteur de ses décisions. De ce fait, il est parvenu tout au long de cette période, à éviter guerres et désastres dans un Proche-Orient en ébullition permanente. Réticent par nature aux grands chambardements, il a réussi le tour de force de ne jamais laisser son pays basculer dans le précipice. 
« L'homme est lucide, commentent Guibal et Salaun, il sait qu'il n'a ni le charisme naturel d'un Nasser, ni le bagout d'un Sadate » (ET, 89). C'est un besogneux, un travailleur. Il est discret, méticuleux, réfléchi. «Une force tranquille, qui apaise les chefs d'Etats étrangers ${ }^{44}$ » $(\mathrm{ET}, 89)$ mais qui l'empêche de s'engager dans la voie des réformes car Moubarak n'a jamais donné, non plus, à l'Egypte « les moyens d'aborder, très haute et bien armée, les défis du XXI ${ }^{\mathrm{e}}$ siècle et de la modernité (ET, 91). Au Caire, cet immobilisme apparaît surtout comme un moyen de verrouiller le pouvoir, en évitant que le pays s'engage dans la voie des réformes. Ce qui vaut à l'Egypte, selon les deux auteurs français, « de se voir très souvent qualifiée de 'dictature molle'. » (ET, 98).

Dictature? Autocratie? Peu importe les appellations même si, quand l'Egypte se mit à bouillir, début janvier 2011, c'est « davantage le second terme qui l'emporte chez les commentateurs de la presse internationale. » (ET, 89). Ce qui importe, comme le dit savamment Alaa el-Aswany, c'est que les longs mandats «entraîne(nt) obligatoirement une inévitable et dangereuse dégénérescence, quelle que soit la personnalité du président ou ses bonnes intentions ». (CRE, 54).

Bien que fondamentalement, la plupart des Egyptiens refusent l'idée de la transmission du pouvoir, que ce soit à Gamal Moubarak ou à n'importe qui d'autre et qu'ils tiennent à leur droit naturel de choisir celui qui les dirigera, c'est cette inévitable dégénérescence qui ancra encore plus les Egyptiens dans leur conviction. Cette dégénérescence consiste essentiellement à la transformation de la république en pouvoir absolu, un pouvoir despotique tout éloigné de la démocratie.

Car la démocratie, elle, obéit à d'autres règles. Dans les pays démocratiques les faits se déroulent d'une tout autre manière: le président de la république occupe ses fonctions pendant huit ans au maximum (deux mandats consécutifs). Il ne peut pas rester un jour de plus à son poste. Un contrôle sévère s'exerce donc sur tout ce qui le concerne lui et sa famille. Le président est choisi par le peuple et lui doit donc son poste. De ce fait, il s'évertue de tenir les promesses sur la base desquelles les électeurs 1'ont choisi. Cela conduit nécessairement à faire appel aux meilleures compétences du pays, qu'il met à profit au service des gens.

${ }^{44}$ Ils y voient le signe d'une rassurante sagesse.

doi :10.12816/0004234 
Quant au président Moubarak, il n'a pas été élu. Après une décennie $^{45}$, il demeura au pouvoir non par le choix des électeurs mais «grâce à la force de l'appareil de sécurité et sa capacité à réprimer les opposants. » (CER, 54). Les référendums commençaient à être truqués, les élections petit à petit fraudées. De ce fait, le président cessa d'accorder une grande importance au peuple. Ce qui lui importe de plus en plus c'était le maintien du pouvoir. Ceux qui l'entourent sont alors recrutés non pas parmi les plus compétents mais plutôt les plus fidèles (à lui non au pays). Le président disposait d'un pouvoir absolu qu'aucune institution ne pouvait contrôler. « Nous ne savons rien de la fortune du président Moubarak et de sa famille, ni quel montant atteint le budget de la présidence de la république, ni quels en sont les différents chapitres. » (CRE, 56).

La politique? A quoi bon s'en mêler? «La génération des jeunes n'y connaît pas grand-chose et ceux qui sont au pouvoir, même s'ils ne sont pas parfaits, savent sûrement mieux qu'elle. Et puis, surtout, que pourrait-il y avoir d'autres ?» $(\mathrm{ET}, 48)$. La majorité des Egyptiens font de même, même ceux qui ne sont pas de la même génération. Ils ont depuis longtemps fait le choix de boycotter les élections. Malgré les tentatives désespérées du régime, le taux de participation n'a jamais dépassé $10 \%$ des électeurs. Il est clair que ce boycott n'a rien de négatif, si oui, pourquoi ces Egyptiens prennent-ils un jour de congé pour venir faire une longue queue afin de pouvoir choisir les membres du nouveau conseil d'administration aux clubs sportifs dont ils sont membres $^{46}$ ? Quand les élections ne sont pas respectables, le boycott « est une position lucide, efficace et juste » (CRE, 110). Il devient le choix approprié, qui interdit au régime de prétendre représenter le peuple qu'il gouverne.

\footnotetext{
${ }^{45}$ Avant que Sadate ne révisât la Constitution cette période de dix ans était presque l'équivalent de deux mandats consécutifs que la constitution défendait de dépasser.

${ }^{46}$ C'est lorsqu'il a été voter au club sportif dont il était membre que Alaa el-Aswany a rencontré une multitude de gens et qu'il a obtenu ces informations. Quand il a demandé à ceux qu'ils connaissaient s'ils participaient aux élections parlementaires la plupart « le dévisageaient d'"un air moqueur et confirmaient qu'ils ne participaient jamais aux élections organisées par le gouvernement, parce qu'elles étaient truquées. Certains ajoutaient qu'ils n'avaient d'ailleurs pas de carte électorale. »(CRE, 111).
} 
Le désintérêt du peuple pousse le régime qui se dirige à grands pas vers la dictature à devenir plus dictatorial encore. On est pris alors dans un cercle vicieux et on finit par se sentir étranger dans son propre pays. Le peuple victime de la dictature passe par des périodes de désespoir national. L'extrémisme naît et les problèmes intercommunautaires interviennent. Pourquoi ? Parce simplement la dictature est contagieuse. Et la tolérance est une faiblesse au milieu de l'extrémisme.

Alaa el-Aswany cite plusieurs exemples pour montrer que le désespoir national et l'extrémisme sont les fils légitimes de la dictature. $\mathrm{Au} \mathrm{XX}^{\mathrm{e}}$ siècle, le désespoir qui s'empara des Egyptiens à cause de l'occupation britannique se transforma rapidement en affreux affrontements interconfessionnels (dans lesquels, comme d'habitude les Britanniques jouèrent un rôle) qui arrivèrent à leur acmé entre 1908 et 1911, mais dès la révolution de 1919, tout le monde s'y fondit au point que certains coptes, comme le père Sergius qui avaient été des inspirateurs de la révolte confessionnelle, devinrent avec la révoltion de grands défenseurs de l'unité nationale.(CRE,206). En Egypte, aujourd'hui, il y a beaucoup de désespoir et de frustrations. Ce sont là des facteurs qui conduisent les Egyptiens aux affrontements confessionnels, exactement comme cela les conduit à la violence, au crime et aux agressions sexuelles.

Quant à l'extrémisme, il est, lui aussi une conséquence immédiate de la dictature. En 1923, une commission formée de certains des plus grands esprits égyptiens fut formée pour élaborer la première constitution égyptienne. Cette commission donna lieu à des échanges politiques et intellectuels de haut niveau au sujet des articles proposés pour la constitution. Des voix se sont en particulier élevées pour demander une représentation proportionnelle des coptes pour garantir dans tous les cas aux coptes un nombre donné de sièges tant au parlement que dans les assemblées locales. Le plus étonnant était que la plupart de ceux qui y étaient opposés étaient des coptes. Ils refusaient de voir dans leurs coreligionnaires une minorité religieuse, mais des citoyens égyptiens qui devaient être appréciés seulement en fonction de critères de compétences. Pourquoi, se demande Alaa el-Aswany était-il difficile de voir un comportement semblable de n'importe quelle secte sous le mandat de Moubarak? La réponse que l'auteur donne est qu'en ce temps-là, "malgré l'occupation britannique, l'Egypte luttait pour fonder un Etat séculier et démocratique assurant l'égalité devant la loi de tous ses citoyens » (CRE, 207). Le régime donnait un exemple de tolérance et invitait implicitement tout le monde à le suivre. La dictature 
et la répression du régime de Moubarak ne permet à personne d'être tolérant.

Le séisme révolutionnaire, cette «lame de fond, invisible, imprévisible » $(\mathrm{ET}, 45)$ a tout emporté sur son passage. Il le fallait. C'était fatal. Car «toutes les conditions d'une révolution se trouvaient (...) réunies. » (CRE, 9) Certes, «il y a 40\% des Egyptiens qui flirtent avec le seuil de la pauvreté. C'est presque $50 \%$ de jeunes au chômage, en particulier des diplômés. Ce système politique fondé sur la corruption, le clientélisme. L'injustice. L'enrichissement inique d'une clique de businessmen, quand une nation entière s'essouffle. Le manque de foi dans les institutions. La quête d'identité d'une nation autrefois glorieuse, aujourd'hui moquée par le reste du monde arabe. Ce rais au pouvoir depuis bientôt trente ans et qui s'y agrippe, préparant son fils à lui succéder. Et cette masse critique, ces 65\% d'Egyptiens au moins de 30 ans, amers, en colère, épuisés avant l'âge.

Le séisme révolutionnaire ne pouvait ne pas avoir lieu. Car l'Egypte n'est pas la Tunisie. C'est l'Egypte. Et on s'étonnait déjà « que la terre du Nil n'ait pas déjà tremblé. » (ET, 45).

C'est ainsi que Alaa el-Aswany d'une part, Claude Guibal et Tangi Salaun d'autre part voient la révolution égyptienne du 25 janvier. De toute cette révolution, nous n'avons étudié, dans les deux ouvrages Chroniques de la révolution égyptienne et l'Egypte de Tahrir que les étincelles de la révolution selon les écrivains. Tandis que les auteurs français voyaient ces étincelles dans l'affaire Khaled Said et les élections législatives de novembre 2010, Alaa el-Aswany soutenait que l'étincelle était le projet de la transmission héréditaire du pouvoir que le régime de Moubarak s'apprêtait à exécuter. Car pour lui, la dictature est la cause de tous des maux. En privant les jeunes de la démocratie on les avait privés de vivre car «la réforme démocratique est le seul chemin vers la réforme économique et la réalisation sociale » $(\mathrm{CER}, 63)$. « La démocratie est la solution ${ }^{47} \gg$. En fait, on avait demandé aux jeunes plus qu'ils ne pouvaient, on les avait condamnés à vivre dans l'ennui, à vivre sans avenir, à se voir étrangers dans leur propre pays pour enfin leur

${ }^{47}$ C'est par cette phrase que Alaa el-Aswany termine chacun de ses articles, à l'exception des plus anciens, rédigés avant qu'il n'ait adopté ce cri de guerre en réaction à «l'islam est la solution» qui servait jusqu'ici à la fois de slogan à l'opposition religieuse et de facile épouvantail à la dictature.

629 
demander de soutenir le fils de l'homme qui les a privés de tout ce dont ils ont rêvé. Le comble de l'injustice. La révolution déclenchée, elle devait continuer. Le régime de Moubarak ne pouvait échapper à sa fin. 


\section{BIBLIOGRAPHIE}

I-Corpus :

1.GUIBAL, Claude \& SALAUN Tangi, L'Egypte de Tahrir, éditions du Seuil, Paris, 2011.

2.El-Aswany, Alaa Chroniques de la révolution égyptienne, traduit de l'arabe, (Egypte) et préfacé par Gilles Gauthier, Actes Sud, Paris, 2011.

\section{II-Ouvrages :}

1.AMINE, Galal, L'Egypte et les Egyptiens sous Moubarak, Dar el-Chourouk, Le Caire, 2011.

2.El-ASWANY, Alaa, Pourquoi les Egyptiens ne se soulèvent-ils pas?, Dar elChourouk, Le Caire, 2011.

3.El-ASWANY, Alaa, La révolution égyptienne s'est-elle trompée?, Dar elChourouk, Le Caire, 2012.

4.EL-BARADEI, Mohamed, Les années de duperie, Dar el-Chourouk, le Caire, 2012.

5.El KHERBAOUI, Sarwat, Le secret du temple, Société Nahdat Misr, Le Caire, 2013.

\section{WEBOGRAPHIE}

1.ABDALLA, Nadine, (chercheuse), Les contestations sociales en Egypte avant et après la Révolution du 25 janvier: réflexions sur l'évolution de leurs formes et de leurs caractéristiques, Arab Forum for Alternatives Studies (AFA), Le

Caire, Doctorante, Institut d'études politiques (IEP), Grenoble. http://www.iemed.org/observatori-fr/arees-danalisi/arxiusadjunts/anuari/med.2012/abdalla_fr.pdf Consulté le 16/11/2013

2.Amnesty international, L'Egypte se soulève Homicides, détentions et tortures pendant la "Révolution du 25 janvier",, mai 2011. http://www.amnesty.org/en/library/asset/MDE12/027/2011/en/7148d6a0d5e3-49e1-af8c-d8494c02ffbd/mde120272011fr.pd Consulté le 16/11/2013

3.BEN NEFISSA,Sarah, Temps électoral, temps révolutionnaire et idiome islamiste en Egypte, http://www.institutgouvernance.org/docs/temps_lectoral_temps_r_volutionnaire.pdf Consulté le 16/11/2013

4.CAILLET, Romain, Les salafis égyptiens à l'heure de la contre-révolution, http://www.religion.info/pdf/2013 10_Caillet.pdf

Consulté le 16/11/2013

5.EL-CHAZLI, Youssef, Une lecture de la "Révolution égyptienne", avril 2011 http://www.sciencespo.fr/ceri/sites/sciencespo.fr.ceri/files/art_yec.pdf Consulté le 16/11/2013

6.El KHAMISSY, Racha, Inversion et révolution: une question de changement d'ordre, université de Ain Chams, Estudios Romanicos, Volumen 21, 2012, pp.79-94

http://revistas.um.es/estudiosromanicos/article/viewFile/166711/144951




\section{P. $81-82$}

7.HEGGAZI, Islam, (professeur adjoint au département des Sciences politiques):

La révolution du 25 janvier et l'avenir de la politique extérieure à l'égard des Etats du bassin du Nil, http://www.sis.gov.eg/Newvr/africa/9fr.pdf

Consulté le 16/11/2013

8.KLAUS, Enrique, (Sciences-Po Rabat (UIR)/ Centre Jacques-Berque (CNRSMAEE): Egypte): la "Révolution du 25 janvier" en contre champ. Chroniques des "comités populaires" d'Al-Manyal au Caire,

http://halshs.archivesouvertes.fr/docs/00/68/22/09/PDF/KLAUS Egypte Tahrir_en contrecha mp 10janv_version dA finitive.pdf Consulté le 16/11/2013

9.PAGES-El KAROUI, Delphine \& VIGNAL, Leila, Les racines de la "révolution du 25 janvier" en Egypte: une réflexion géographique, 2011 http://echogeo.revues.org/12627?lang=en Consulté le 16/11/2013

10.STADNICKI, Roman, Comprendre Le Caire ...pour comprendre la révolution égyptienne?

http://hal.inria.fr/docs/00/68/09/16/PDF/Comprendre_Le_Caire_recension_St adnicki.pdf a.Consulté le 16/11/2013

11.VANNETZEL, Marie, L'émergence d'une mobilisation multisectorielle en Egypte: des prémisses contextuelles aux basculements situationnels, http://www.sciencespo.fr/ceri/sites/sciencespo.fr.ceri/files/art_mv_0.pdf Consulté le 16/11/2013

12.Egypte: Deux ans après la révolution, la protection des droits fondamentaux des citoyens est toujours inexistante, mise à jour le 23 janvier 2013.

http://www.fidh.org/fr/maghreb-moyen-orient/Egypte,129/Egypte-Deux-ans-apresla-12790

Consulté le 16/11/2013

13.Les révoltes et les révolutions dans le monde: qu'en reste-t-il? L'esprit révolutionnaire: contagion dans le monde arabe. http://www.cms.fss.ulaval.ca/recherche/upload/hei/fichiers/regardcritique avril2011_web.pdf Consulté le 16/11/2013

14.Paroles de jeunes. Reportage.

http://www.imf.org/external/pubs/ft/fandd/fre/2012/03/pdf/voices.pdf Consulté le 16/11/2013

15.Passages: art et artistes égyptiens durant et après la Révolution http://www.prohelvetia.ch/fileadmin/user upload/customers/prohelvetia/ Publikationen/Passagen/pdf/fr/Passagen_F58_geschuetzt.pdf Consulté le 16/11/2013

16.Une Révolution trahie: rapport spécial http://www.equaltimes.org/wp-content/uploads/2013/06/Egypt-FRFinal.pdf Consulté le 16/11/2013 
مجلة الدراسات التربوية والأسانية _كلية التربية ـ جامعة دمنهور ـ المجلد الخامس العدد(4)- الجزءوالثلث- لسنة 2013

633 